\title{
German Medical Informatics Initiative: Unlocking Data for Research and Health Care
}

\author{
Stefanie Gehring ${ }^{1 *}$; René Eulenfeld ${ }^{2 *}$ \\ ${ }^{1}$ DLR Project Management Agency, Bonn, Germany; \\ ${ }^{2}$ Project Management Jülich, Jülich, Germany
}

\begin{abstract}
Summary
This article is part of the Focus Theme of Methods of Information in Medicine on the German Medical Informatics Initiative. The Medical Informatics Initiative of the German Federal Ministry of Education and Research will make use of the potential of digitalization in the field of medicine in Germany. The aim is to improve the possibilities for medical research and patient care through innova-
\end{abstract}

Correspondence to:

Stefanie Gehring, PhD

DLR Project Management Agency

Heinrich-Konen-Straße 1

53227 Bonn

Germany

E-mail: Stefanie.Gehring@dlr.de

* DLR Project Management Agency and the Project Management Jülich manage the Medical Informatics Initiative on behalf of the German Federal Ministry of Education and Research. tive IT solutions. In an initial step, data integration centres will be set up at university hospitals to ensure the technical and organizational conditions necessary for multi-site exchange of data between health care and clinical and biomedical research. The Federal Ministry of Education and Research will provide a total of around EUR 150 million for this initiative over the next four years.

Methods Inf Med 2018; 57(0pen 1): e46-e49 https://doi.org/10.3414/ME18-13-0001

received: February 2, 2018 accepted: February 9, 2018

\section{Introduction}

Recent advances in biomedical research, such as massive parallel sequencing and imaging technologies, have led to an enormous increase in the generation of biomedical data. At the same time, the volume of data in digital form in patient care and clinical research is growing fast. The integration and analysis of these data can be of enormous benefit for medical care.

Yet, the usability of this wealth of data is still seriously limited due to a variety of problems such as data heterogeneity, lack of structured data and fragmentation of sources. Data are typically stored where they are collected and exist in diverse formats determined by the original purpose of acquisition. For example, routine data from patient care are not primarily designed to be queried, extracted and used for research purposes. Moreover, access to such data and their use are mainly restricted to those professionals directly involved in treatment. In Germany, documentation is separated according to inpatient and outpatient care and a common electronic health record is not yet in place. Systematic collections of research data such as registries, biobanks, trial databases or collections of "omics" data also often follow their own standards and are rarely interconnected.

In order to overcome these obstacles, the German Federal Ministry of Education and Research launched the "Medical Informatics" funding scheme, which is also known as the Medical Informatics Initi- ative, in November 2015 [1, 2]. An overview of the funding scheme and the current status of the funding activities are set out below.

The aim of the Medical Informatics Initiative is to improve both research opportunities and patient care through innovative IT solutions which enable the collaborative use of data by the research community and the health care system across institutions and locations. It is a strategic programme, combining the development of IT infrastructure, scientific research projects, as well as the promotion of junior researchers and education in medical informatics.

University hospitals are the initial focal point of activities in the Medical Informatics Initiative due to the close connection between research and patient care at such institutions. Technical and organizational infrastructures will be set up at university hospitals to make clinical and research data comprehensively available to the medical sector. Data protection and data security will be a major issue when linking and integrating vast amounts of personalized data. Appropriate solutions for consent management will have to be developed and implemented to protect the data of the subjects concerned. New conceptual frameworks will have to be drawn up to provide the foundations for the joint use of clearly defined data and a shared understanding of the requirements for data models and data types. Implementing Semantic and syntactic interoperability are consequently one of the most important facets of the (funding) activities.

The advances in medical informatics are expected to provide benefits for a variety of stakeholders: For example, custom-tailored therapy for patients will be expanded and 
physicians will profit from improved data sets supporting clinical decision making. Researchers will be able to use data otherwise unavailable to them, which will also facilitate the recruitment of patients for clinical trials. In addition, young data scientists and other clinical/research personnel will be trained to ensure the innovative strength, competitiveness and connectivity of research and health care in the future.

\section{The Modular Design of the Funding Scheme Ensures Flexibility}

The German Medical Informatics Initiative adopts a tiered approach and is modular in design, allowing for flexibility to adapt to results obtained, as well as future technical advances and requirements ( $\triangleright$ Figure 1).

The main funding module involves consortia consisting of university hospitals plus further academic and industry partners, as described below. Supporting activities which, for example, involve a National Steering Committee and its coordination office support the cooperation between the consortia. Supplementary funding activities are envisaged and can be implemented on demand, for instance to address ethical, legal and social aspects of digitalization in health care and data sharing or to foster projects for international collaborations.

The funding scheme is being implemented in three successive phases. It started with a nine-month conceptual phase in 2016/2017, followed by a four-year development and networking phase from 2018 to 2021 during which the core groundwork will be carried out in all the funding modules. A subsequent third phase is planned for the consolidation and further development of successful solutions.

\section{Four Consortia Move into the Key Phase of the Initiative}

As of January 2018, the solutions developed in the preceding conceptual phase are being implemented and tested in the development and networking phase. The four most promising of the seven consortia that initially entered the conceptual phase were selected to enter this phase on the basis of the recommendations of an international evaluation board. The four successful consortia are called: DIFUTURE, HiGHmed, MIRACUM, and SMITH. Each of these four will now receive funding of around EUR 30 million for the next four years.

University hospitals form the core of the consortia. Overall, 17 of the 33 German university hospitals are part of these four consortia so far. The university hospitals of the remaining three consortia of the conceptual phase are invited to join the four consortia in 2018/2019, meaning that nearly all German university hospitals can partake in the consortia. Additional funding of up to EUR 30 million in total is available for their participation. Besides university hospitals, the four consortia have further partners from academia and industry, e.g. research institutions such as the German Cancer Research Centre at Heidelberg, higher education institutions, private clinics, and companies from the IT, pharmaceutical, biotechnology or medical technology sectors ( $\triangleright$ Figure 2).

\section{Data Integration Centres, Use Cases and Junior Research Groups as Building Blocks}

The main task of the consortia is the development and implementation of technical and organizational solutions to enable the exchange and use of data from research and health care across institutions and sites. The key structural element is the establishment of data integration centres at each university hospital. Their role is to provide access to local, previously unlinked

\section{Medical Informatics Funding Scheme}
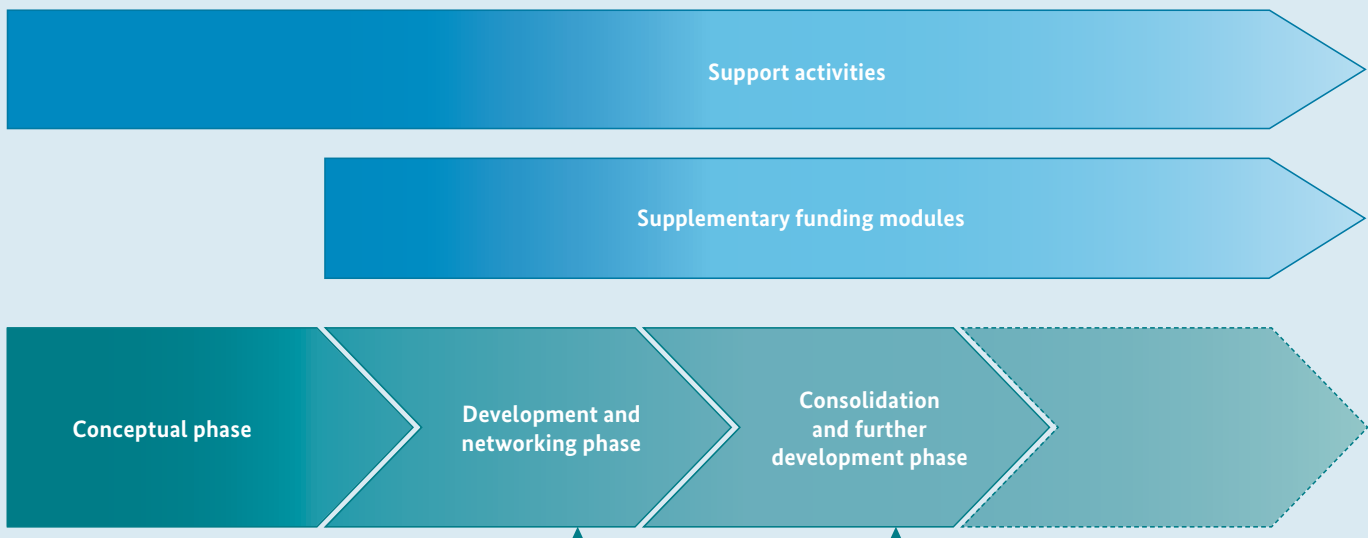

Supplementary funding modules

2016-2017
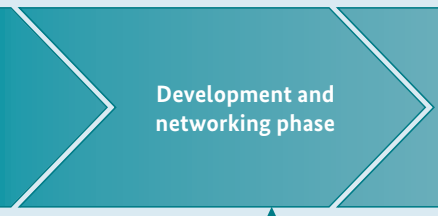

2018-2021

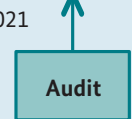

2022-2025

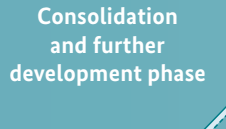

ase
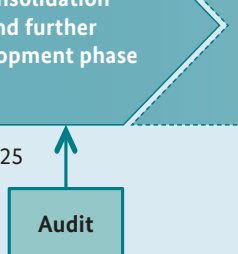

Figure 1 Overview of the funding scheme with its parallel modules and successive funding phases. Modules from bottom to top: consortia; supplementary funding modules; and support activities, such as the coordination office of the National Steering Committee. Graphic adapted from Medical Informatics Funding Scheme [1]. 


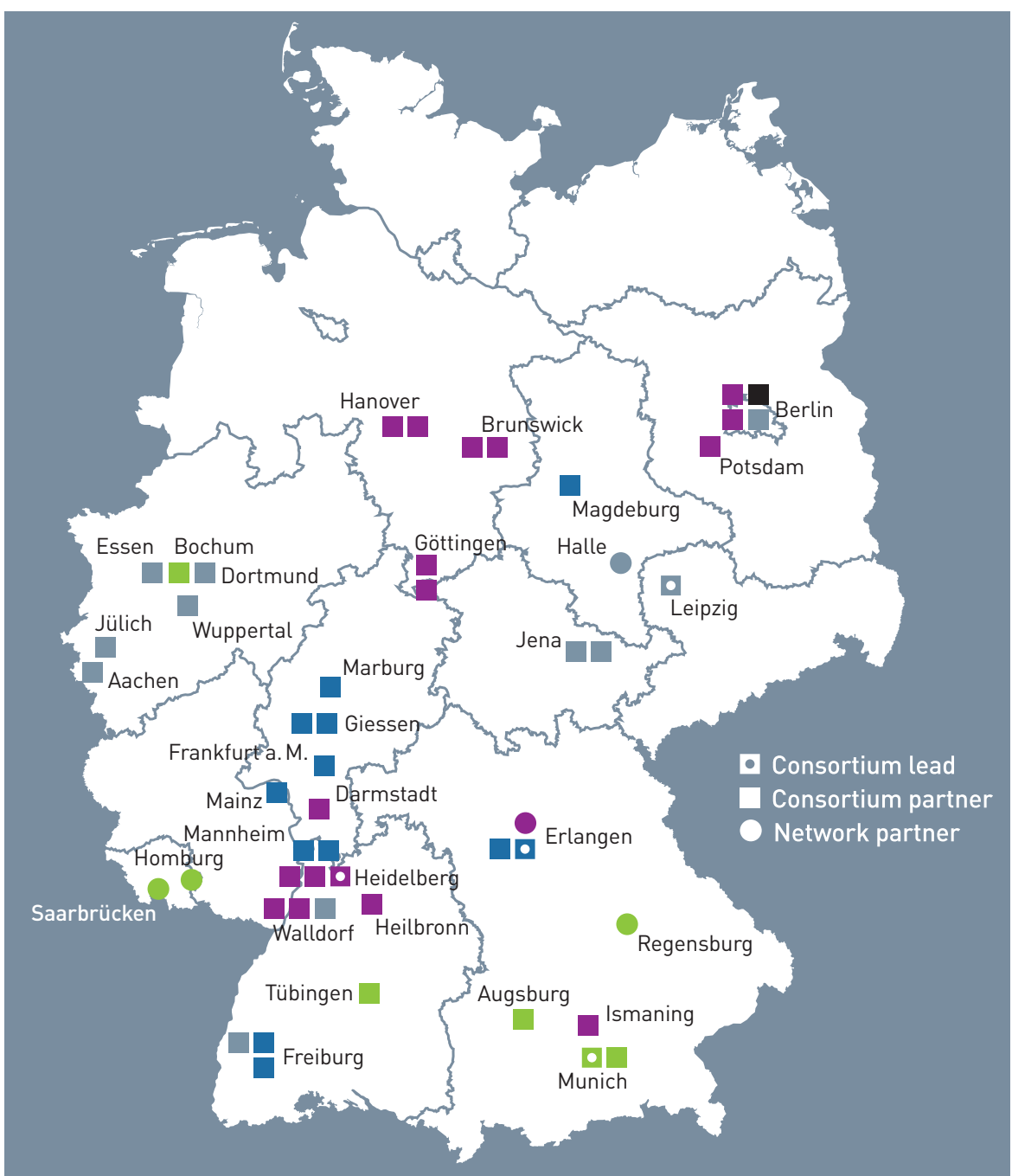

Figure 2 Location of consortia partners. All the partners in the consortia DIFUTURE (green), HiGHmed (magenta), MIRACUM (blue), SMITH (grey) and the coordination office (black) are shown with their location in Germany (as of Dec. 2017). 11 further university hospitals can join them during 2018/2019. Figure kindly provided by Malte Belau for TMF e.V.

and thus inaccessible data and create interfaces to exchange data with other university hospitals. Among other tasks, the data integration centres will assure compliance with data protection regulations, and train and support users. The developed solutions have to be internationally compatible. One of the responsibilities of the consortia's advisory boards is to ensure such compatibility.

The second important task of the consortia in this phase is the development and implementation of IT solutions for specific applications (use cases) to exemplify the usability of the data integration centres and demonstrate the benefits of data sharing. Prototype use cases will address a variety each university and the German Federal Ministry of Education and Research. As an incentive to establish new professorships in medical informatics, all participating higher education institutions in the four consortia can apply for parallel funding of junior research groups. As of now, around 30 new professorships in medical informatics or related areas have been announced and several of them are already in the appointment process.

\section{National Steering Committee: Prevention of Stand-alone Solutions}

Coordination between the consortia is vital to prevent stand-alone solutions and to pave the way for national roll-outs. A National Steering Committee has already been established to promote cross-consortia collaboration in the conceptual phase. All the consortia are represented in this Committee, which identifies and addresses the challenges and needs for common action. Dedicated working groups jointly discuss and prepare requirements for common approaches, concept papers and text templates for final agreement by the National Steering Committee. A range of papers on strategic aspects and central challenges were prepared during the conceptual phase. These include a common agreement on specifications of core data sets, harmonized metadata sets, and measures to achieve interoperability within and between consortia. Organizational and scientific support for the National Steering Committee and its working groups is provided by a coordination office.

\section{The Medical Informatics Initiative as an Important Milestone towards a Digitally Networked Health Care System}

What are the next steps? Six months before the end of the development and networking phase, an external audit will assess whether the consortia have successfully implemented technical and organizational 
solutions for data sharing and created added value for research and patient care. Depending on these results, a third phase is planned for the consolidation and further development of promising solutions. These will then be rolled out to additional university hospitals and possibly other hospitals and research institutions. Ultimately, it is intended that successful solutions will be implemented nationwide.

Currently, the digitally networked health care system is still a vision. But advances in medical informatics will help to accomplish this goal and the initiative provides an important milestone in this direction. Patients can benefit greatly from the wealth of data unlocked. The ultimate aim is that at any given point within the health care system, decisions can be taken on the basis of the full scope of the relevant data and medical knowledge available from research and the health care system, all while respecting the right of individuals to maintain control over their personal data.

The Medical Informatics Initiative promises to transform clinical research and patient care - and yet many challenges still lie ahead before a digitally connected health care system can become reality. In order to overcome these challenges, it is important that all relevant stakeholders work together. At the Digital Summit of the German Federal Government in June 2017, the Federal Ministry of Education and Research and the Federal Ministry of Health announced plans to establish a dialogue platform for digital health where relevant players from civil society, research, health care, and industry will be brought together to discuss the possibilities and challenges of digitalization and big data in the health system.

\section{References}

1. Medical Informatics Funding Scheme [cited 2018 Jan 21]. Available from: https://www.bmbf.de/pub/ Medical_Informatics_Funding_Scheme.pdf.

2. Regulations for funding for the conceptual phase and the development and networking phase of the "Medical Informatics" funding scheme [cited 2018 Jan 21]. Available from: http://www.dlr.de/pt/Port aldata/45/Resources/Dokumente/GF/Announcement-English-Version.pdf. 